\title{
ESTRATEGIAS PARA OPTIMIZAR EL MANEJO FARMACOLÓGICO EN EL ADULTO MAYOR
}

\author{
Paola Casas-Vásquez ${ }^{1, a}$, Pedro Ortiz-Saavedra1,2,b, Eduardo Penny-Montenegro ${ }^{1,3, c}$
}

\begin{abstract}
RESUMEN
El envejecimiento de la población tiene como consecuencia incrementos en la prevalencia de enfermedades crónicas y de multimorbilidad, en el consumo de múltiples fármacos y los problemas relacionados con los mismos. Se reporta que más del $50 \%$ de todos los medicamentos expendidos son consumidos por adultos mayores; más del $80 \%$ de las personas adultas mayores toman al menos una medicación diaria y $75 \%$ no informa a su médico que usa tratamientos no convencionales. Prescribir de forma apropiada en el adulto mayor es un trabajo difícil que requiere considerar un balance entre los riesgos y beneficios de las medicinas indicadas, las cuales suelen no tener una evidencia clara de su eficacia, dada la poca representatividad de la población adulta mayor en los ensayos clínicos randomizados sobre los cuales se basan las guías clínicas para el manejo de las enfermedades crónicas, condiciones altamente prevalentes en la población adulta mayor. Sin embargo, existen directrices y recomendaciones generales que deben de aplicarse de forma integral, apoyados por un equipo multidisciplinario, toda vez que se realice un manejo farmacológico en el adulto mayor. En este artículo, nos enfocamos en promover la prescripción adecuada sobre la base de recomendaciones basadas en evidencia para disminuir la medicación inapropiada, la polifarmacia y la automedicación.
\end{abstract}

Palabras clave: adulto mayor, farmacoterapia, polifarmacia, automedicación (fuente: DeCS BIREME).

\section{STRATEGIES TO OPTIMIZE PHARMACOLOGICAL MANAGEMENT IN OLDER ADULTS}

\begin{abstract}
Population aging increases the prevalence of chronic and multiple morbid illnesses and increases the consumption of multiple medications and related problems accordingly. It is reported that $>50 \%$ of dispensed medications are consumed by older adults; in fact, $>80 \%$ of older adults take at least one medication daily and $75 \%$ do not inform their doctor that they are using unconventional treatments. Appropriate medication prescription to older adults is challenging and it requires the consideration of risks and benefits of the indicated medications, for which clear evidence of their efficacy is often lacking due to the limited inclusion of elderly populations in randomized clinical trials on which clinical guides are based for chronic disease management. However, general guidelines and recommendations must be comprehensively implemented and supported by a multidisciplinary team whenever pharmacological management is provided for older adults. Here we focus on promoting proper prescriptions according to evidence-based recommendations to reduce inappropriate prescriptions, polypharmacy, and self-medication
\end{abstract}

Key words: elderly, pharmacotherapy, polypharmacy, self-medication (source: MESH NLM).

\section{INTRODUCCIÓN}

La transición demográfica ya no es exclusiva de los países desarrollados, y este acelerado proceso de envejecimiento de la población trae consecuencias como incrementos en la prevalencia de enfermedades crónicas, de multimorbilidad, en el consumo de múltiples fármacos y, por ende, en los problemas relacionados con los mismos. El manejo farmacológico, en general, es un punto álgido en salud pública y de mayor preocupación en la población adulta mayor, debido a que es un grupo etario particular y susceptible, que requiere competencias específicas del personal de salud para reducir el elevado riesgo de eventos adversos asociados al inadecuado uso de fármacos ${ }^{(1,2)}$.

Los factores involucrados son múltiples, desde los cambios relacionados a la edad en la farmacocinética y farmacodinámica, que se acentúan ante la presencia de

\footnotetext{
Instituto de Gerontología, Universidad Peruana Cayetano Heredia. Lima, Perú.

Facultad de Medicina, Universidad Peruana Cayetano Heredia. Lima, Perú.

Academia Nacional de Medicina. Lima, Perú.

Médico geriatra; ${ }^{\mathrm{b}}$ médico internista, magíster en Medicina; ${ }^{\mathrm{c}}$ médico internista y geriatra, magíster en Medicina

Recibido: 07/03/2016 Aprobado:04/05/2016
}

Citar como: Casas-Vásquez P, Ortiz-Saavedra P, Penny-Montenegro E. Estrategias para optimizar el manejo farmacológico en el adulto mayor. Rev Peru Med Exp Salud Publica. 2016;33(2)335-41. doi: 10.17843/rpmesp.2016.332.2153 
fragilidad; el llamado factor receta, que incluye la buena escritura; la comprensión adecuada y las indicaciones claras por parte del prescriptor, así como la selección y dispensación del fármaco en múltiples escenarios como asilos, hospitales, centros de atención primaria y farmacias de la propia comunidad ${ }^{(2,3,4)}$.

Por ello, la Organización Mundial de la Salud (OMS) define el concepto de uso racional de medicamentos al hecho de recibir un medicamento apropiado para la necesidad clínica del paciente, en las dosis necesarias a sus requerimientos, por un tiempo adecuado y a un menor costo para él y la comunidad ${ }^{(5)}$. Sin embargo, el problema de los medicamentos en los adultos mayores siguen siendo frecuentes; se reporta que un tercio de medicamentos son prescritos para personas mayores de 65 años, más del $50 \%$ de todos los medicamentos expendidos son consumidos por adultos mayores, más del $80 \%$ de las personas adultas mayores toman al menos una medicación diaria y $75 \%$ no informa a su médico que usa tratamientos no convencionales, incluidas hierbas medicinales ${ }^{(6,7)}$.

En Perú, los estudios relacionados a fármacos son limitados; un estudio realizado en el $2005^{(8)}$, analizó las características de la prescripción farmacológica en adultos mayores hospitalizados en el Servicio de Medicina del Hospital Nacional Cayetano Heredia, se evaluó 170 pacientes encontrándose 1535 prescripciones farmacológicas de 121 fármacos diferentes y 295 prescripciones potencialmente inapropiadas, que representaron el $19,2 \%$ del total; se halló una correlación significativa entre la prescripción inapropiada con la polifarmacia y el número de diagnósticos al ingreso. Por otro lado, en un estudio prospectivo en el Hospital Nacional Arzobispo Loayza, en pacientes admitidos a emergencia ${ }^{(9)}$, encontraron que las hospitalizaciones por eventos adversos a medicamentos en adultos mayores representaron el 5,5\% del total de los ingresos, $20,6 \%$ de este grupo tuvieron eventos adversos a medicamentos previos y $82,5 \%$ de casos se dieron a dosis adecuadas. Oscanoa ${ }^{(10)}$ encuentra que los problemas relacionados con medicamentos en adultos mayores son frecuentes al momento de ser hospitalizados en el Servicio de Geriatría del Hospital Guillermo Almenara (EsSALUD) con un 45,8\% de prescripción inapropiada, $63 \%$ de falta de adherencia y $24 \%$ de reacciones adversas a fármacos.

En esta revisión nos enfocamos en la promoción de la prescripción adecuada con recomendaciones para evitar una mala indicación basadas en evidencia actual, sobre todo disminuyendo la medicación inapropiada, la automedicación y la polifarmacia.

\section{REDUCIR LA PRESCRIPCIÓN INADECUADA DE MEDICAMENTOS EN EL ADULTO MAYOR}

La prescripción inadecuada comprende diversos errores como elegir fármacos que no corresponden al tratamiento más eficaz, indicar medicinas que interactúen entre sí, recetar dosis incorrectas o, incluso, el uso de una vía de administración inapropiada. De ahí la importancia de medir la calidad de la prescripción farmacológica que, si bien a la fecha no cuenta con una "prueba de oro" existen diversos instrumentos, entre los más usados en investigaciones fármacoepidemiológicas se encuentran los criterios de Beers; los criterios de STOP/START; los criterios de McLeod de práctica inadecuada en la prescripción a adultos mayores; los criterios de Laroche sobre medicación potencialmente inadecuada en población mayor de 75 años; el ACOVE (Assessing Care Of Vulnerable Elders), y el índice de uso adecuado de medicamentos (Medication Appropriateness Index o MAI) ${ }^{(11-13)}$. Se han desarrollado nuevos grupos de criterios para la detección de medicación inapropiada en pacientes mayores, la lista PRISCUS (14); el algoritmo de buena práctica paliativa geriátrica ${ }^{(15)}$, y el IPET (Improved Prescribing in the Elderly Tool) ${ }^{(16)}$

Basados en ellos, y dependiendo de la ubicación, la prescripción inapropiada puede fluctuar desde un $14 \%$ utilizando los criterios de Beers en la comunidad, un $40 \%$ en consulta hospitalaria de geriatría según criterios STOPP (Screening Tool of Older Person's Prescriptions) o un $92 \%$ en estudios hospitalarios según los criterios MAl (Medication Appropriateness Index). Regueiro et al. (17), evaluaron el uso de medicamentos y la prescripción inadecuada en 215 adultos mayores de la comunidad en ciudad de La Plata, encontrando frecuencias de medicación potencialmente inapropiada en $25,5 \%$, $31,9 \%$ y $30 \%$ según los criterios de Beers, lista PRISCUS y criterios STOPP. Kersten et al. (18) también evaluaron a 232 adultos mayores de 75 años, procedentes de la comunidad y con comorbilidad, admitidos a emergencia, encontrando que la hospitalización no cambió la frecuencia de polifarmacia o el uso de medicación potencialmente inapropiada, hubo mejor manejo de medicación por equipos geriátricos multidisciplinario y no hubo impacto en las mediciones clínicas evaluadas (mental, funcional, fragilidad y velocidad de la marcha).

Estos instrumentos evalúan el proceso y el resultado en base a valoraciones implícitas (basados en el juicio) y explícitas (basadas en criterios); sin embargo, estas herramientas tienen algunas desventajas, sobre todo al extrapolarlos a realidades como las de nuestro país donde las investigaciones son escasas, existe carencia de medicamentos y donde generalmente las prioridades de las intervenciones farmacológicas van dirigidas a enfermedades transmisibles, sugiriendo que en este contexto sería más útil una lista de medicamentos costo efectivo y seguros para enfermedades crónicas prevalentes en adultos mayores más que una lista a evitarse (11). Esto, de alguna manera, se podría contemplar en los criterios de STOPP/START, instrumento europeo que trata de mejorar algunas limitaciones de los criterios de Beers, en el que, además de enumerar medicamentos inapropiados (STOPP), tiene otro listado de medicamentos que deben prescribirse en patologías concretas (START), basadas en evidencias disponibles respecto a su uso. 
Oscanoa (19) propone una lista de chequeo para el uso seguro de medicamentos en el adulto mayor, la cual está constituida por diez pasos validados, con la finalidad de prevenir la sobreprescripción (prescribir más medicamentos de lo clínicamente necesarios), disprescripción (prescripción incorrecta de un fármaco que tiene una indicación clínica) y la infraprescripción (omisión de la intervención farmacológica de probada efectividad).

Tanzi ${ }^{(20)}$ sugiere, además, algunas pautas basadas en dificultades comunes: aplicar herramientas como criterios de Beers o el índice de adecuación de la medicación (MAl), reducir al máximo el riesgo de caídas, evitar los anticolinérgicos, evitar el uso de antipsicóticos de segunda generación en psicosis mediada por demencia, poner énfasis en dosis bajas de anticoagulación oral, vigilar los medicamentos de venta sin prescripción, verificar necesidad de apoyo al manipular envases de medicamentos, y alertar sobre existencia de medicamentos homófonos u homógrafos.

\section{REDUCIR LA POLIFARMACIA EN EL ADULTO MAYOR}

Se define polifarmacia al consumo simultáneo de más de tres medicamentos, situación muy común en adultos mayores dada la coexistencia de múltiples comorbilidades. Actualmente, la definición de polifarmacia posee un espectro que va más allá del número y que incluye la pertinencia de la prescripción, es decir, un adulto mayor puede recibir múltiples fármacos si cada uno de ellos posee una eficacia demostrada para las condiciones que presenta, al margen de cuántos sean. Lo contrario ocurre cuando el número de fármacos es elevado gracias a medicinas que son totalmente evitables dada su probada ineficacia, su limitado beneficio para un adulto mayor o su alto riesgo de desarrollar eventos adversos, a esta situación se le ha llamado polifarmacia inadecuada ${ }^{(21,22)}$.

La polifarmacia en los adultos mayores, especialmente en aquellos frágiles, está asociada de forma proporcional al deterioro funcional tanto físico como social, a la disminución de la adherencia a fármacos esenciales, a elevados costos tanto para los pacientes como para los propios servicios de salud y al incremento del riesgo de eventos adversos a medicamentos, interacciones medicamentosas, delirio, caídas, hospitalización y muerte ${ }^{(2,3)}$.

La frecuencia de la polifarmacia varía según el ámbito donde se estudie y la definición utilizada. En los Estados Unidos de América, la población adulta mayor, en general, está expuesta a polifarmacia entre 14 a $37 \%$, siendo mayor al $40 \%$ en residencias geriátricas. En Europa, la frecuencia varía entre 12 al $80 \%$, cifra más alta en residencias geriátricas ${ }^{(23-25)}$. En nuestro país, las cifras son similares, cerca del $45 \%$ de los adultos mayores atendidos ambulatoriamente presentan polifarmacia ${ }^{(26)}$.

La gran mayoría de los adultos mayores son portadores de enfermedades crónicas, aquellas situaciones clínicas que tienen duración prolongada, que necesitan atención médica continuada y, a su vez, tienen la capacidad de limitar las actividades de la vida diaria; las prevalencias son más altas en países en desarrollo. La mayoría de los adultos mayores tiene más de una patología en simultaneo, para lo cual, usualmente, reciben múltiples fármacos $\mathrm{y}$, por ende, están expuestos a un mayor riesgo de presentar eventos adversos, interacciones medicamentosas y falta de adherencia ${ }^{(27)}$.

La forma de evaluar la prescripción farmacológica, ya sea en términos de polifarmacia y de si es adecuada o no, puede realizarse mediante escalas validadas y ya presentadas previamente. Sin embargo, estos instrumentos comparten la falencia de ser muy específicos para algunos fármacos y condiciones, de no ser generalizables en todos los escenarios y países con prácticas de prescripción muy particulares por cada región y finalmente, de que su utilidad no se ha podido asociar con resultados de salud como reducir la morbilidad, mortalidad, las admisiones hospitalarias y la calidad de vida del adulto mayor ${ }^{(28,29)}$.

Un problema frecuente que se asocia al incremento en el número de fármacos resulta de la denominada cascada de prescripción, situación que se suscita asumiendo erróneamente que un nuevo síntoma debe ser manejado con un nuevo fármaco y no descartar que se deba a uno de los fármacos recibidos y que, por lo tanto, debiera ser suspendido (30). Situaciones clínicas han sido ampliamente descritas y deben ser tomadas en cuenta de forma permanente al manejar múltiples fármacos y ante la aparición de nuevos síntomas.

Se ha publicado recientemente una revisión Cochrane ${ }^{(31)}$ para determinar la efectividad de las intervenciones, solas o en combinación, en reducir la polifarmacia inadecuada en adultos mayores habiéndose encontrando beneficio significativo en su frecuencia; sin embargo, el efecto clínico y directo sobre la salud del adulto mayor aún es desconocido en términos de calidad de vida, hospitalizaciones, reducción de eventos adversos y morbimortalidad. Se revisaron doce estudios, donde todos incluyeron un trabajo multidisciplinario compuesto por farmacéuticos, enfermeras y personal médico asistencial en diversos escenarios, y las medidas comprendían estrategias de revisión de medicinas, recomendaciones terapéuticas, monitoreo de medicación, educación al paciente y a los profesionales de la salud, conferencias multidisciplinarias, desarrollo de listas de problemas y protocolos de manejo. Un solo estudio usó un sistema computarizado de soporte 
de decisiones. Todas mostraron beneficio en reducir la prescripción inapropiada, mas no hubo certeza en si estas medidas tuvieron mejoría clínica significativa.

Se han publicado múltiples recomendaciones, muchas de ellas abordando la intervención de manera multidimensional, multidisciplinaria y contemplando las preferencias y particularidades del paciente y su familia. Una aproximación es el desarrollo de los principios para una prescripción prudente desarrollada por Schiff et al. ${ }^{(32)}$, quienes utilizan los estudios científicos junto con el sentido común para listar una serie de principios que aseguran una prescripción adecuada. Si bien reconoce que cada una de las recomendaciones por separado es eficaz individualmente, el seguimiento de las recomendaciones en global es la que asegura el éxito terapéutico. Se describen a continuación aquellas con demostrada eficacia en estudios clínicos.

Revisar la medicación siempre que se tiene contacto con el paciente en los momentos de evaluación ya sea en consulta ambulatoria u hospitalización, así como detener la administración de medicinas innecesarias son medidas eficaces y seguras que minimizarían la polifarmacia y los eventos adversos ${ }^{(33)}$.

Aquellas intervenciones que se apoyan en sistemas computarizados de soporte de decisiones involucrando al equipo multidisciplinario también han tenido un efecto positivo en reducir la polifarmacia, por lo que se recomienda el uso de sistemas informáticos de ayuda para la toma de decisiones, que incluya alertas inmediatas en caso de prescripciones redundantes, interacciones farmacológicas y prescripciones potencialmente inadecuadas. Esto hace más eficaz la comunicación entre médicos y farmacéuticos, y pueden alertar de posibles errores, duplicidades e interacciones. Un estudio ha demostrado que las prescripciones mejoran usando una base de datos de fármacos en adultos mayores ${ }^{(34,35)}$.

La educación tanto del personal de salud como de los pacientes y sus familiares tiene un efecto positivo en prescribir apropiadamente la medicación ${ }^{(36)}$. Se deben conocer los cambios relacionados a la edad en la farmacocinética y farmacodinamia así como las interacciones entre la fragilidad y las medicinas. Los pacientes y familiares deben tomar parte en las decisiones que se toman en relación al tratamiento pues la educación ha demostrado mejorar las tasas de adherencia al tratamiento así como reducir los reingresos hospitalarios ${ }^{(37)}$.

Es importante el compromiso de todo el equipo multidisciplinario trabajando en forma coordinada, lo que ha demostrado ser eficaz en mejorar la calidad de la prescripción en el adulto mayor. El equipo multidisciplinario que incluye enfermeras, farmacéuticos y el médico a cargo, deben revisar la lista de medicamentos y estar atentos ante los problemas potenciales derivados del uso continuo de la medicación (38).

\section{PREVENIR LA AUTOMEDICACIÓN EN EL ADULTO MAYOR}

La OMS considera la automedicación como responsable cuando hay un uso adecuado de la misma, ya que se conoce los síntomas de la enfermedad y el medicamento para contrarrestarla, lo que no ocurre con la auto prescripción (automedicación no responsable) en que la conducta es conseguir medicamentos de venta bajo receta médica sin contar con ella. Dahir et al. (39), en base a la experiencia argentina, citan como causas de automedicación a algunos factores como: la medicalización de la vida (situaciones cotidianas que no requieren de medicación), percepción de la necesidad de tomar fármacos y creer por experiencias personales o de terceros la eficacia del medicamento, la influencia de los medios de comunicación, el autocuidado excesivo y el rol vertical del médico en la prescripción.

La automedicación es un problema que afecta a todos los grupos etarios; un estudio desarrollado en Lima encuentra que la automedicación es cuatro veces más frecuente en adultos mayores comparado con poblaciones más jóvenes en el estrato socioeconómico alto ${ }^{(40)}$. Si bien se ha demostrado asociación entre el nivel educativo de quienes se automedican con este grave problema, afrontarlo y eliminarlo supone cambios en la regulación del sistema de salud y abordar el compromiso de educar a la sociedad en general y a los propios profesionales de la salud, por parte del estado y las instituciones comprometidas en salud, para evitar consecuencias en esta etapa de la vida. Asimismo, las entidades reguladoras del expendio de medicinas deberán tener un mejor sistema de vigilancia para reducir la venta de medicinas sin receta médica y regular la participación de los diferentes actores de la cadena de la medicación: productor, prescriptor, dispensador y consumidor.

\section{RECOMENDACIONES PARA OPTIMIZAR EL MANEJO FARMACOLÓGICO EN EL ADULTO MAYOR}

Elaborar una prescripción apropiada en el adulto mayor es un trabajo difícil que requiere considerar un balance entre los riesgos y beneficios de las medicinas indicadas, las cuales muchas veces no tienen una evidencia clara de su eficacia en este grupo etario, especialmente en el grupo de adultos mayores frágiles y polimedicados, dada su poca representatividad en los ensayos clínicos randomizados sin que se pueda extrapolar los resultados de poblaciones jóvenes ${ }^{(41,42)}$. Adicionalmente, el manejo de las enfermedades crónicas, altamente prevalente en la población adulta mayor y presentes de forma simultánea, se suele basar en recomendaciones 
plasmadas como guías clínicas las cuales suelen ser específicas para cada enfermedad individual y extrapoladas de estudios con pacientes usualmente seleccionados sin comorbilidades ni polifarmacia, lo cual dificulta su aplicación en adultos mayores ya que puede elevar el riesgo de deterioro y calidad de vida, si son aplicables de forma rígida sin considerar las particularidades del adulto mayor ${ }^{(43-46)}$.

Sin embargo, existen directrices y recomendaciones generales que deben de aplicarse de forma integral y apoyados por un equipo multidisciplinario, toda vez que se realice un manejo farmacológico en el adulto mayor. Resulta necesario enfocar más esfuerzos de todas las instituciones educativas de salud en enseñar a los médicos en entrenamiento y a los médicos de atención primaria sobre las particularidades que hacen que el adulto mayor sea más propenso a desarrollar complicaciones derivadas de una mala prescripción ${ }^{(47,48)}$.

De las recomendaciones publicadas y resumidas en la literatura se pueden listar las siguientes directrices para una prescripción adecuada (Tabla 1).
También es importante educar a los adultos mayores y sus cuidadores para contribuir en asegurar una atención diferenciada, por lo que se recomienda:

1. Hacer una lista para el médico, de todos los medicamentos usados en forma rutinaria u ocasionalmente.

2. Informar al médico sobre cualquier condición que pueda afectar su capacidad para tomar ciertos medicamentos, como alergias, infartos, hipertensión, enfermedad cardiaca severa o problemas hepáticos, renales o pulmonares.

3. Preguntar o aclarar cualquier duda que tenga.

4. Siempre que sea posible, solicitar informaciones por escrito.

5. Organizar sus medicamentos para facilitar su localización, identificación y administración.

6. Revisar periódicamente sus medicamentos y descartar aquellos que estén vencidos.

7. Hablar con su médico respecto a costos.

Contribuciones de autoría: PCV, POS y EPM han participado conjuntamente en la concepción del artículo, su redacción y aprobación de la versión final.

Fuentes de financiamiento: autofinanciado.

Conflictos de interés: los autores declaran no tener conflictos de interés.

Tabla 1. Directrices con ejemplos para una prescripción adecuada

\section{Directrices}

1. Evaluar y definir claramente el problema del paciente.

2. Especificar el objetivo terapéutico y seleccionar el medicamento más adecuado.

3. Usar el menor número de fármacos indispensables.

4. Evaluar constantemente si la terapia indicada es eficaz y necesaria.

5. Iniciar el tratamiento con los datos apropiados y detallados

6. Promover el uso de terapias no farmacológicas antes de iniciar un fármaco.

7. Establecer objetivos claros y viables considerando expectativa y calidad de vida.

8. Conocer la farmacología, interacciones y eventos adversos de los fármacos.

9. Considerar la aparición de un nuevo síntoma como un posible evento adverso.

10. Proporcionar información, instrucciones y advertencias claras.

11. Evitar la prescripción de medicamentos de baja utilidad terapéutica.

12. Usar herramientas tecnológicas para reducir errores de prescripción.

\section{Ejemplo}

En pacientes con cuadro clínico de demencia, se deberá buscar causas reversibles, como lo es el déficit de vitamina B12 o hipotiroidismo ${ }^{(49)}$

Pacientes adultos mayores con hipertensión arterial tienen por objetivo presiones menores a $150 / 90 \mathrm{mmHg}$, con el fármaco que mejor toleren ${ }^{(50)}$.

En pacientes hipotiroideos con síntomas depresivos, el tratamiento hormonal podría mejorar la depresión y así evitar más fármacos ${ }^{(51)}$

El adulto mayor diabético debe clasificarse en frágil o no frágil regularmente en sus controles, de ello dependerá sus objetivos de Hba1c y el tratamiento evitando el riesgo de hipoglicemia. ${ }^{(52)}$

En adultos mayores con fibrilación auricular no valvular se requiere de una valoración geriátrica integral para el inicio de anticoagulación oral ${ }^{(53)}$.

Recomendar adoptar medidas de higiene de sueño en pacientes adultos mayores con insomnio ${ }^{(54)}$.

En pacientes diabéticos considerados en etapa terminal o al final de la vida el objetivo terapéutico es evitar la hipoglicemia. Se considera retirar la terapia en forma progresiva ${ }^{(52)}$.

Evitar el uso de antinflamatorios no esteroideos en adultos mayores ya que pueden inhibir efecto de antihipertensivos, agravar la insuficiencia cardiaca, incrementar riesgo de hiperkalemia, nefrotoxicidad y sangrado en combinación con otros fármacos ${ }^{(30)}$.

Los medicamentos antiparkinsonianos pueden provocar hipotension ortostatica y delirio y ello conllevar a caídas ${ }^{(55)}$.

Verificar que tanto el paciente como el cuidador o persona a cargo del paciente entienda las indicaciones, escribir con letra clara, legible y términos sencillos. Advertir de eventos adversos y signos de alarma.

Evitar prescripción de glucosamina en pacientes con osteoartrosis ${ }^{(56)}$.

El uso de alertas vía teléfono o correo electrónico para identificar a pacientes con falla renal y prevenir eventos adversos reducen el riesgo de eventos adversos ${ }^{(57)}$. 


\section{REFERENCIAS BIBLIOGRÁFICAS}

1. Cho S, Law S, Tandon V, Kumi K, Pfuma E, Abernethy DR. Geriatric drug evaluation: where are we now and where should we be in the future? Arch Intern Med.2011;171(10):937-40. doi: 10.1001/archinternmed.2011.152.

2. Varela L. Principios de geriatría y gerontología. 2a Ed: Lima; Universidad Peruana Cayetano Heredia; 2011. pp 251-261.

3. Klarin I, Wimo A, Fastbom J. The association of inappropriate drug use with hospitalization and mortality: a population-based study of the very old. Drugs Aging. 2005;22(1):69-82.

4. Wauters M, Elseviers M, Vaes B, Degrysse J, Stichele R, Christiaens $\mathrm{T}$, et al. Mortality, Hospitalisation, Institutionalisation in communitydwelling oldest old: The impact of medication. Arch Gerontol Geriatr. 2016;65:9-16. doi: 10.1016/j. archger.2016.02.009.

5. Organización Mundial de la Salud. Promoción del uso racional de medicamentos: componentes centrales. Ginebra: OMS; 2002.

6. Shorr R, Hoth AB, Rawls N, editores. Drugs for the geriatric patient. Philadelphia: Saunders Elsevier; 2007.

7. Hayes BD, Klein-Schwarzt W, Barrueto F Jr. Polypharmacy and the geriatric patient. Clin Geriatr Med. 2007;23(2):371-90.

8. Córdova V, Chávez H, Varela L, Ortiz P, Mendez F. Características de la prescripción farmacológica en pacientes adultos mayores hospitalizados. Diagnostico. 2005;44(4):151-9.

9. Ortiz P, Juárez M, Valdivia, Varela L. Hospitalización por eventos adversos relacionados a medicamentos en adultos mayores. Rev Soc Peru Med Interna. 2009;22(2):53-6.

10. Oscanoa TJ. Diagnóstico de problemas relacionados con medicamentos en adultos mayores al ser hospitalizados. Rev Peru Med Exp Salud Pública. 2011;28(2):256-63.

11. Oscanoa T, Castañeda B. Calidad de la prescripción farmacológica en geriatría. Instrumentos de evaluación. $2^{\mathrm{a}} \mathrm{Ed}$. Lima: CONCYTEC; 2012.

12. Fick DM, Cooper JW, Wade WE, Waller JL, Maclean JR, Beers MH.
Updating the Beers criteria for potentially inappropriate medication use in older adults: results of a US consensus panel of experts. Arch Intern Med. 2003;163(22):2716-24.

13. Gallagher P, Ryan C, Byrne S, Kennedy J, O'Mahony D. STOPP (Screening Tool of Older Person's Prescriptions) and START (Screening Tool to Alert doctors to Right Treatment). Consensus validation. Int $\mathrm{J}$ Clin Pharmacol Ther. 2008;46(2):72-83.

14. Holt S, Schmiedl S, Thurmann PA. Potentially inappropriate medications in the elderly: the PRISCUS list. Dtsch Arztebl Int. 2010;107(31-32):543-51. doi: 10.3238/arztebl.2010.0543.

15. Garfinkel D, Mangin D. Feasibility study of a systematic approach for discontinuation of multiple medications in older adults: addressing polypharmacy. Arch Intern Med. 2010;170(18):1648-54. doi: 10.1001/ archinternmed.2010.355.

16. Naugler CT, Brymer C, Stolee P, Arcese ZA. Development and validation of an improving prescribing in the elderly tool. Can J Clin Pharmacol. 2000;7(2):103-7.

17. Regueiro M, Mady N, Cañás M, Farina HO, Nagal P. Uso de medicamentos en adultos mayores no institucionalizados. Rev Peru Med Exp Salud Pública. 2011;28(4):643-47.

18. Kersten H, Hvidsten LT, Gloersen G, Willer TB, Wang-Hansen MS. Clinical impact of potentially inappropriate medications during hospitalization of acutely ill older patient with multimorbidity. Scand J Prim Health Care 2015;33(4):243-51. doi: $10.3109 / 02813432.2015 .1084766$.

19. Oscanoa TJ. Uso seguro de medicamentos en adultos mayores: una lista de chequeo. Rev Peru Med Exp Salud Publica. 2013;30(2):320-5.

20. Tanzi MG. Ten tips for counseling older adults. Pharmacy today [Internet]. 2011 [cited 2016, May 11]; 17(11). Disponible en: http://www. pharmacist.com/AM/template redirect. $\mathrm{cfm}$ ? template $=/ \mathrm{CM} /$ contentdisplay. cfm\&ContentID $=273622$

21. Aronson JK. Rational prescribing, appropriate prescribing. $\mathrm{Br} \mathrm{J}$ Clin Pharmacol. 2004;57(3):229-30.
22. Steinman MA, Landefeld CS, Rosenthal GE, Berthenthal D, Sen S, Kaboli PJ. Polypharmacy and prescribing quality in older people. J Am Geriatr Soc. 2006;54(10):1516-23

23. Gavilán Moral E, Morales Suárez-Varela MT, Hoyos Esteban JA, Pérez Suanes AM. Polimedicación y prescripción de fármacos inadecuados en pacientes ancianos inmovilizados que viven en la comunidad. Aten Primaria. 2006;38(9):476-82.

24. Slabaugh SL, Maio V, Templin M, Abouzaid S. Prevalence and risk of polypharmacy among the elderly in an outpatient setting: a retrospective cohort study in the EmiliaRomagna region, Italy. Drugs Aging. 2010;27(12):1019-28

25. Hovstadius B, Hovstadius K, Astrand B, Petersson G. Increasing polypharmacy - an individual-based study of the Swedish population 2005-2008. BMC Clin Pharmacol. 2010;10:16. doi: 10.1186/1472-6904-10-16.

26. Casas P, Varela L, Tello T, Ortiz P, Chavez H. Perfil clínico del adulto mayor atendido ambulatoriamente en un hospital general. Rev Med Hered. 2012;23(4):229-34.

27. Barnett K, MercerSW, Norbury M, Watt G, Wyke S, Guthrie B. Epidemiology of multimorbidity and implications for health care, research, and medical education: a cross-sectional study. Lancet. 2012;380(9836):37-43. doi: $10.1016 /$ S0140-6736(12)60240-2.

28. Yong TY, Khow KSF. Prescribing appropriately in frail older people. Healthy Aging Research. 2015;4:21. doi:10.12715/har.2015.4.21.

29. Murray MD, Callahan CM. Improving medication use for older adults: an integrated research agenda. Ann Intern Med. 2003;139(5 Pt 2):425-9.

30. Rochon PA, Jurwitz JH. Optimising drug treatment for elderly people: the prescribing cascade. BMJ. 1997;315(7115):1096-9.

31. Cooper JA, Cadogan CA, Patterson SM, Kerese N, Bradley MC, Ryan $\mathrm{C}$, et al. Interventions to improve the appropriate use of polypharmacy in older people: a Cochrane systematic review. BMJ Open. 2015;5(12):e009235. doi:10.1136/ bmjopen-2015-009235. 
32. Schiff GD, Galanter WL, Duhig J, Lodolce AE, Koronkowski MJ, Lambert BL. Principles of conservative prescribing. Arch Intern Med. 2011;171(16):1433-40. doi: 10.1001/ archinternmed.2011.256.

33. Scott IA, Hilmer SN, Reeve E, Potter K, Le Couteur D, Rigby D. Reducing inappropriate polypharmacy: the process of deprescribing. JAMA Intern Med. 2015;175(5):827-34. doi: 10.1001/jamainternmed.2015.0324.

34. Shrank WH, Polinski JM, Avorn J. Quality indicators for medication use in vulnerable elders. J Am Geriatr Soc. 2007; 55 Suppl 2:S373-82. doi: 10.1111/j.1532-5415.2007.01345.x.

35. Monane M, Matthias DM, Nagle BA, Kelly MA. Improving prescribing patterns for the elderly through an online drug utilization review intervention: a system linking the physician, pharmacist, and computer. JAMA 1998;280(14):1249-52. doi:10.1001/jama.280.14.1249.

36. Jackson SH, Mangoni AA, Batty GM. Optimization of drug prescribing. $\mathrm{Br}$ J Clin Pharmacol. 2004;57(3):231-6. doi: 10.1046/j.1365-2125.2003.02018.x

37. Peterson AM, Takiya L, Finley R. Metaanalysis of trials of interventions to improve medication adherence. Am J Health Syst Pharm. 2003;60(7):65765.

38. Crotty M, Halbert J, Rowett D, Giles L, Birks R, Williams $\mathrm{H}$, et al. An outreach geriatric medication advisory service in residential aged care: a randomised controlled trial of case conferencing. Age Ageing. 2004;33(6):612-7. doi: 10.1093/ageing/afh213.

39. Dahir C, Hernandorena C, Chagas L, Mackern K, Varela V, Alonso I. Automedicación: un determinante en el uso racional de medicamentos. Evid Act Pract Amb. 2015;18(2):46-9.

40. Mestanza F, Pamo O. Estudio muestral del consumo de medicamentos y automedicación en Lima Metropolitana. Rev Med Hered. 1992;3(3):101-8.

41. Fortin M, Dionne J, Pinho G, Gignac J, Almirall J, Lapointe L. Randomized controlled trials: do they have external validity for patients with multiple comorbidities? Ann Fam Med. 2006;4(2):104-8.

42. Boyd CM, Darer J, Boult C, Fried LP, Boult L, Wu AW. Clinical practice guidelines and quality of care for older patients with multiple comorbid diseases: implications for pay for performance. JAMA. 2005;294(6):716-24. doi:10.1001/ jama.294.6.716.

43. Tinetti ME, Bogardus ST Jr, Agostini JV. Potential pitfalls of diseasespecific guidelines for patients with multiple conditions. N Engl J Med. 2004;351(27):2870-4. doi: 10.1056/ NEJMsb042458.

44. Mutasingwa DR, Ge H, Upshur RE. How applicable are clinical practice guidelines to elderly patients with comorbidities? Can Fam Physician. 2011;57(7):e253-62.

45. Cox L, Kloseck M, Crilly R, McWilliam C, Diachun L. Underrepresentation of individuals 80 years of age and older in chronic disease clinical practice guidelines. Can Fam Physician. 2011;57(7):e263-9.

46. Lugtenberg $M$, Burgers JS, Clancy C, Westert GP, Schneider EC. Current guidelines have limited applicability to patients with comorbid conditions: a systematic analysis of evidence based guidelines. PLoS One. 2011;6(10):e25987. doi: 10.1371/ journal.pone.0025987.

47. Pollock M, Bazaldua OV, Dobbie AE. Appropriate prescribing of medications: an eight-step approach. Am Fam Physician. 2007;75(2):231-6.

48. Cullinan S, Fleming A, O'Mahony D, Ryan C, O'Sullivan D, Gallagher $\mathrm{P}$, et al. Doctors' perspectives on the barriers to appropriate prescribing in older hospitalized patients: a qualitative study. Br J Clin Pharmacol 2015;79(5):860-9. doi: 10.1111/ bcp. 12555 .

49. Custodio N, Escobar J, Altamirano J, Bendezú C, Montesinos R, Lira $\mathrm{D}$, et al. Demencia por deficiencia de vitamina b12: ¿siempre hay respuesta terapéutica? Acta Med Per. 2011;28(4):221-23.

50. James P, Oparil S, Cárter BL, Cushman WC, Dennison-Himmelfarb C, Handler J, et al. 2014 evidence-based guideline for the management of high blood pressure in adults: report from the panel members appointed to the Eighth Joint National Committee (JNC 8). JAMA. 2014;311(5):507-20. doi:10.1001/jama.2013.284427.

51. Ugalde O. Guía clínica para el tratamiento de los trastornos psicogeriátricos. Ed. $S$ Berenzon, J Del Bosque, J Alfaro, ME MedinaMora. México: Instituto Nacional de Psiquiatría. (Serie: Guías Clínicas para la Atención de Trastornos Mentales). México: Instituto Nacional de Psiquiatría Ramón de La Fuente Muñiz México; 2010.

52. American Diabetes Association. Older adults. Sec. 10. In Standards of Medical Care in Diabetes-2016. Diabetes Care. 2016;39(Suppl 1):S81-S85. doi: $10.2337 / \mathrm{dc} 16-\mathrm{S} 013$.

53. Veiga Fernández F, Malfeito Jiménez Mdel R, Barros Cerviño SM, Magariños Losada Mdel M. Oral anticogulation for non-valvular atrial fibrilation in the elderly. Rev Esp Geriatr Gerontol. 2015;50(3):134-42. doi: $10.1016 / j$. regg.2015.01.005.

54. Medina-Chávez JH, Fuentes Alexandro SA, Gil-Palafox IB, Adame-Galván L, Solis-Lam F, Sánchez-Herrera LY, et al. Guía de practica Clinica diagnóstico y tratamiento del insomnio en el adulto mayor. Rev Med inst Méx Seguro Soc. 2014;52(1):108-19.

55. Peralta-Pedrero ML, Valdivia-Ibarra FJ, Hernández-Manzano M, Medina Beltrán GR, Cordero-Guillén MA, Baca-Zúñiga J, et al. Guía de práctica clínica. Prescripción farmacológica en el adulto mayor. Rev Med Inst Méx Seguro Soc. 2013;51(2):228-39.

56. Wandel S, Jüni P, Tendal B, Nüesch E, Villiger PM, Welton NJ, et al. Effects of glucosamine, chondroitin, or placebo in patients with osteoarthritis of hip or knee: network meta-analysis. BMJ. 2010;341:c4675. doi: 10.1136/bmj. c4675.

57. Joosten H, Drion I, Boogerd KJ, van der Pijl EV, Slingerland RJ, Slaets JP, et al. Optimizing drug prescribing and dispensing in subjects at risk for drug errors due to renal impairment: improving drug safety in primary healthcare by low eGFR alerts.BMJOpen. 2013;3(1):pii.e002068. doi:10.1136/bmjopen-2012-002068.

Correspondencia: Paola Casas Vásquez

Dirección: Instituto de Gerontología, Universidad Peruana Cayetano Heredia

Teléfono: (511) 3190007

Correoelectrónico: igero@oficinas-upch.pe 